\title{
Clinical use of sglt-2 inhibitors for the management of type 2 diabetes in a patient centered medical home
}

\begin{abstract}
Purpose: To provide current data regarding the efficacy and tolerability of sodium-glucose cotransporter-2 (SGLT-2) inhibitors in type 2 diabetes mellitus (T2DM) patients at a large patient-centered medical home (PCMH) Multi-Specialty Physicians' Group.

Methods: A retrospective observational study that took place at a PCMH located in Buffalo, NY. Eighty-one adults aged 18-95years old with T2DM on a SGLT-2 inhibitor (canagliflozin, dapagliflozin, or empaglifozin) for a minimum of 3 months. Change in hemoglobin $\mathrm{A} 1 \mathrm{c}(\mathrm{HbAlc})$ in addition to the percentage of patients achieving a $\mathrm{HbA} 1 \mathrm{c}$ level below $7 \%$. Changes in weight, changes in systolic (SBP) and diastolic (DBP) blood pressures, percentage of patients reporting adverse effects of therapy, and percentage of patients with an estimated glomerular filtration rate (eGFR) $<60 \mathrm{~mL} / \mathrm{min} / 1.73 \%$.
\end{abstract}

Results: 81 patients met inclusion criteria. Mean $\mathrm{HbAlc}$ was significantly reduced by $1.04 \%$ after 3 months of therapy with $25.9 \%$ more patients achieving target $\mathrm{HbA} 1 \mathrm{c}$ below $7 \%$. A significant reduction was also observed in weight by an average of 7.33 pounds and in both SBP by $10 \mathrm{mmHg}$ and DBP by $4 \mathrm{mmHg}$. Adverse effects were reported in $14.8 \%$ of patients with only 3 out of the 10 patients discontinuing therapy due to intolerance to side effects. $18.5 \%$ of patients had a decline in eGFR less than $60 \mathrm{~mL} / \mathrm{min} / 1.73 \%$.

Conclusion: SGLT-2 inhibitors exhibited tolerability and efficacy in T2DM patients who need to treat multiple aspects of their disease. This class of diabetic agents has the capability of maintaining control of hyperglycemia as well as blood pressure and weight, with minimal incidence of side effects.

Keywords:sglt-2 inhibitors, PCMH, diabetes mellitus, efficacy, tolerability
Volume 3 Issue 8 - 2016

\author{
Nicole P Albanese, ${ }^{2}$ Elisa Torres, ' Ashley \\ Woodruff,' Andre Lopez ${ }^{2}$ \\ 'University at Buffalo, School of Pharmacy and Pharmaceutical \\ Sciences, USA \\ ²Buffalo Medical Group, USA
}

\begin{abstract}
Correspondence: Nicole Paolini Albanese, University at Buffalo, School of Pharmacy and Pharmaceutical Sciences, 209 Kapoor Hall, Buffalo, NY 14203, Tel 716 645 39I 5, Fax 716829 609 3, Email npaolini@buffalo.edu
\end{abstract}

Received: December 08, 2016 | Published: December 12, 2016
Abbreviations: $\mathrm{PCMH}$, patient centered medical home; SGLT-2 Inhibitors, sodium glucose Cotransport-2 Inhibitors; T2DM, type 2 diabetes; A1c, hemoglobin a1c; SBP, systolic blood pressure; DBP, diastolic blood pressure; Egfr, estimated glomerular filtration rate; $\mathrm{CDC}$, centers for disease control; ADA, american diabetes association; AACE, american academy of clinical endocrinology; SU, sulfonylureas; TZD, thiazolidinediones; DPP-4 Inhibitor, dipeptidyl peptidase 4; EMR, electronic medical record; CAD, coronary artery disease; PAD, peripheral artery disease; T1DM, type 1 diabetes; ACE, angiotensin-converting-enzyme

\section{Introduction}

The National Diabetes Statistics Report, published by the Centers for Disease Control and Prevention (CDC), reports that 29.1million Americans over 20years old were estimated to have diabetes. This includes those that may be undiagnosed accounting for about 8.1million adults. ${ }^{1}$ Type 2 diabetes mellitus (T2DM) specifically affects $90 \%$ to $95 \%$ of diagnosed patients, characterized by insulin resistance due to pancreatic beta cell dysfunction. Diabetes becomes more complicated in its association with other serious health conditions such as cardiovascular disease, obesity, and kidney failure increasing the risk of death by $50 \%$ compared to people without diabetes. ${ }^{1}$ Uncontrolled glycemic levels produce a vicious cycle of deteriorating beta cell function and further worsening of hyperglycemia. ${ }^{2}$ The likelihood for treatment failure is heightened as beta cells become less responsive to changes in insulin sensitivity.
Majority of patients need combination therapy of metformin and one or more anti-hyperglycemic agents to maintain target $\mathrm{HbAlc}$ levels below $7 \% .{ }^{1,3}$ Improved glycemic control has been shown to reduce the progression of microvascular complications as well as macrovascular complications. ${ }^{4}$ Guidelines for the American Diabetes Association (ADA) Standards of Care were recently updated to include sodiumglucose co-transporter 2 (SGLT-2) inhibitors as an appropriate adjunct therapy to Metformin. ${ }^{5}$ The American Association of Clinical Endocrinologists (AACE) and American College of Endocrinology (ACE) consensus statement recommends using SGLT-2 inhibitors as first line therapy. ${ }^{6}$ Other agents include sulfonylureas (SU), thiazolidinediones (TZD), dipeptidyl peptidase 4 (DPP-4) inhibitors, glucagon-like peptide-1 (GLP-1) receptor agonists, and basal insulin. Though these treatment options have been available longer, they are restricted by side effects such as weight gain, hypoglycemia, and cardiovascular risk. ${ }^{7}$ Canagliflozin was the first SGLT-2 inhibitor approved in the United States, followed by dapagliflozin and most recently, empagliflozin. Their distinctive site of action in the kidneys and their insulin independent mechanism sets these drugs apart from previous pharmacologic targets in diabetic therapy allowing them to work well synergistically with other agents while avoiding common side effects. ${ }^{8,9}$ Renal filtration involves regulating plasma glucose concentration. In normal adults, $90 \%$ of glucose is filtered through the kidneys daily, then reabsorbed in the proximal tubule by the SGLT-2 transporters, thus resulting in a negligent amount of glucose excreted into the urine. By inhibiting SGLT-2 transporters, glucosuria occurs, 
lowering plasma glucose levels. In addition to decreasing $\mathrm{HbAlc}$, the renal mechanism has also promoted weight loss, improved systolic blood pressure, and minimized hypoglycemic events. Due to the fact that many T2DM patients are overweight or obese, weight loss mechanisms are highly desirable when choosing therapy. Patients with T2DM have a greater risk of developing cardiovascular and health complications. ${ }^{1,4}$ Although not proven to directly modify beta cell function, improvement in blood glucose with an SGLT-2 inhibitor has a favorable effect on beta cell function. ${ }^{9,10}$ SGLT-2 inhibitors offer a new role in T2DM therapy for patients who have not achieved $\mathrm{HbA1c}$, blood pressure, and/or weight loss goals and have experienced negative side effects from other diabetes drug classes. The progressive nature of diabetes and its growing incidence necessitates effective treatment that will benefit multiple disease components.

\section{Materials and methods}

The objective of this study was to provide current data regarding the efficacy and tolerability of SGLT-2 inhibitors in T2DM patients at a large patient-centered medical home $(\mathrm{PCMH})$ Multi-Specialty Physicians' Group. Patient data was collected from the electronic medical record (EMR) and analyzed separately to determine that all inclusion criteria were met. Findings were documented in a spreadsheet that organized patient demographics (age, gender, and race), pertinent co-morbidities (hypertension, obesity, coronary artery disease (CAD) or peripheral artery disease (PAD), and renal impairment), medication history (other diabetic agents and hypertension therapy), laboratory results ( $\mathrm{HbAlc}, \mathrm{SCr}$, blood pressure, calculated $\mathrm{BMI}$, and weight) and reported side effects associated specifically with SGLT-2 inhibitors (hypotension, decline in eGFR $<45 \mathrm{~mL} / \mathrm{min} / 1.73 \mathrm{~m}^{2}$, nasopharyngitis, urinary tract infections, and genital mycotic infections).

Candidates for the study were 18 to 95 years old diagnosed with T2DM and had documentation of at least three months of SGLT-2 inhibitor use. Exclusion criteria included diagnosis of type 1 diabetes (T1DM) or gestational diabetes, pregnancy, declined renal function defined as an eGFR $<45 \mathrm{~mL} / \mathrm{min} / 1.73 \mathrm{~m}^{2}$, patients who changed diabetic (except insulin) and/or hypertension therapy during the study period, patients who underwent weight loss surgery or had a weight loss medication in their profile. The primary endpoint for evaluating the efficacy of SGLT-2 inhibitors was the change in HbAlc from baseline. This endpoint also assessed the difference in the percentage of patients who achieved optimal $\mathrm{HbAlc}$ goal $<7 \%$ after the addition of SGLT-2 therapy. Secondary endpoints evaluating additional therapeutic effects and the safety profile of SGLT-2 inhibitors were changes in weight, changes in systolic blood pressure (SBP) and diastolic blood pressure (DBP), and percentage of patients who declined in renal function to an eGFR $<60 \mathrm{~mL} / \mathrm{min} / 1.73 \%$ compared to baseline. Descriptive statistics were used to summarize demographic and anthropometric characteristics of the study groups. Data normality was assessed using D'Agostino \& Pearson normality test. Paired t-test was used to evaluate continuous variables. McNemar's test was used to evaluate nominal and dichotomous data. Statistical significance was determined by a $P$ value $<0.05$. The University at Buffalo Institutional Review Board deemed this study to be exempt from IRB approval on May 14, 2015.

\section{Results}

An EMR search identified 137 candidates for the study. Using prespecified exclusion criteria, a total of 56 patients were excluded with the majority being due to lack of sufficient data or follow-up (Figure
1). Eighty-one patients were eligible to participate in the study with demographics and baseline characteristics displayed in Table 1. The study population was predominantly Caucasian, middle aged to older adults with obesity. Additional diabetes and hypertension therapy is also shown in Table 1 . The most common diabetic agents utilized at baseline were metformin, DPP-4 inhibitors, and sulfonylureas, which accounted for $72 \%, 43 \%$, and $33 \%$ of the patient population respectively. Baseline co-morbidities (Table 1) included patients with history of hypertension $(\mathrm{n}=70)$, renal insufficiency $(\mathrm{n}=24)$, and obesity $(n=60)$. Within our study population, $75 \%$ of patients were on either an angiotensin-converting-enzyme (ACE) inhibitor or an angiotensin receptor blocker (ARB).

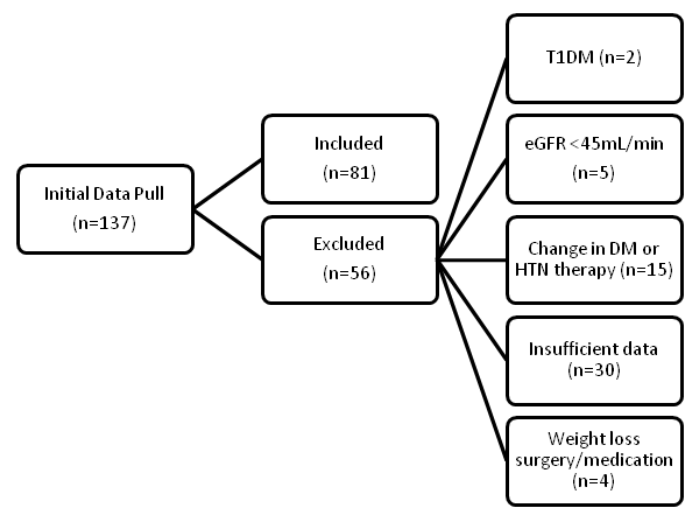

Figure I Pre-specified exclusion criteria.

TIDM=Type I Diabetes Mellitus; DM=Diabetes Mellitus; HTN=Hypertension.

Table I Demographical Data

\begin{tabular}{ll}
\hline Demographic data(n=8 I) & \\
\hline Mean age (years) & $6 \mathrm{I}$ \\
Gender, n(\%) & $57(70.4)$ \\
Male & \\
SGLT-2, n(\%) & \\
Canagliflozin & $69(85.2)$ \\
Dapagliflozin & $10(12.3)$ \\
Empagliflozin & $2(2.5)$ \\
Mean duration of SGLT-2 use (months) & 5 \\
Race, n(\%) & \\
Caucasian & $64(79)$ \\
African American & $9(\mathrm{I} 1 . \mathrm{I})$ \\
Asian & $3(3.7)$ \\
Hispanic & $\mathrm{I}(\mathrm{I} .2)$ \\
Native American/Alaskan & $3(3.7)$ \\
Unknown & $\mathrm{I}(\mathrm{I} .2)$ \\
Comorbidities, n(\%) & \\
Obesity & $60(74.1)$ \\
Hypertension & $70(86.4)$ \\
CAD & \\
& \\
\hline
\end{tabular}


Table continued..

\begin{tabular}{|c|c|}
\hline \multicolumn{2}{|l|}{ Demographic data $(n=8 I)$} \\
\hline PAD & $4(4.9)$ \\
\hline Renal insufficiency & $24(29.6)$ \\
\hline \multicolumn{2}{|l|}{ Concurrent diabetes medications, n(\%) } \\
\hline Metformin & $58(71.6)$ \\
\hline Sulfonylurea & $27(33.3)$ \\
\hline Alpha-glucosidase inhibitor & I (I.2) \\
\hline Thiazolidinedione & $7(8.6)$ \\
\hline Dipeptidyl Peptidase-4 (DPP-4) Inhibitor & $35(43.2)$ \\
\hline Glucagon-like Peptide-I (GLP-I) Agonist & $18(22.2)$ \\
\hline Insulin & $25(30.9)$ \\
\hline \multicolumn{2}{|c|}{ Concurrent blood pressure medications, n(\%) } \\
\hline $\begin{array}{l}\text { Angiotensin-Converting Enzyme (ACE)/ } \\
\text { Angiotensin Receptor Blocker (ARB) }\end{array}$ & $61(75.3)$ \\
\hline Beta Blocker (BB) & $31(38.3)$ \\
\hline Calcium Channel Blocker (CCB) & $22(27.2)$ \\
\hline Diuretic & $28(34.6)$ \\
\hline Other & $2(2.5)$ \\
\hline
\end{tabular}

Table 2 Primary and secondary endpoints

\begin{tabular}{|c|c|c|c|}
\hline Characteristic $(n=8 I)$ & Baseline & 3months & P-value \\
\hline $\mathrm{HbAIc}(\%)$, mean \pm SD & $8.67 \pm 1.49$ & $7.63 \pm 1.31$ & $<0.0001$ \\
\hline $\mathrm{HbAlc}<7 \%, \mathrm{n}(\%)$ & $7(8.6)$ & $28(34.6)$ & $<0.0001$ \\
\hline Weight (lb), mean \pm SD & $233 \pm 48$ & $226 \pm 50$ & $<0.0001$ \\
\hline $\mathrm{SBP}(\mathrm{mmHg})$, mean $\pm \mathrm{SD}$ & $134 \pm 15$ & $124 \pm 11$ & $<0.0001$ \\
\hline $\mathrm{DBP}(\mathrm{mmHg})$, mean \pm SD & $77 \pm \underline{+8}$ & $73+8$ & 0.0021 \\
\hline eGFR <60mL/min/l.73\%, n(\%) & $5(6.2)$ & $15(18.5)$ & 0.0063 \\
\hline
\end{tabular}

$\mathrm{SBP}=$ systolic blood pressure; $\mathrm{DBP}=$ diastolic blood pressure; eGFR=estimated glomerular filtration rate

Table 3 Side effects reported

\begin{tabular}{ll}
\hline Side effect reported $(\mathbf{n = 8}$ I) & $\mathbf{n}(\%)$ \\
\hline eGFR decline $(<45 \mathrm{~mL} / \mathrm{min} / \mathrm{I} .73 \%)$ & $5(6.2 \%)$ \\
Nasopharyngitis & $3(3.7 \%)$ \\
Genital Mycotic Infection & $2(2.5 \%)$ \\
Hypotension & $\mathrm{I}(\mathrm{I} .2 \%)$ \\
Urinary Tract Infection & $\mathrm{I}(\mathrm{I} .2 \%)$ \\
\hline
\end{tabular}

eGFR=estimated glomerular filtration rate

Of the SGLT-2 inhibitors, canagliflozin was used by $85 \%$ with only $10 \%$ accounting for dapagliflozin users and $2 \%$ accounting for empagliflozin users. Canagliflozin was often initiated at $100 \mathrm{mg}$ daily and titrated to a daily dose of $300 \mathrm{mg}$ if the patient sufficiently tolerated use. Mean duration of SGLT-2 inhibitor therapy was 5months. Summary of results from study primaryand secondary endpoints can be viewed in Table 2. In at least 3months of use, mean HbA1c was significantly reduced by $1.04 \%(<0.0001)$ with 28 patients $(\mathrm{P}<0.0001)$ reaching an $\mathrm{HbA} 1 \mathrm{c}$ goal of $<7 \%$, which was 21 more patients than at baseline. All secondary endpoints reached statistical significance. Participants lost an average of 7.33 pounds $(\mathrm{P}<0.0001)$. Blood pressure reduction was seen in both $\mathrm{SBP}(-10 \mathrm{mmHg} ; \mathrm{P}<0.0001))$ and DBP $(-4 \mathrm{mmHg}$; $\mathrm{P}=0.0021)$. SGLT-2 inhibitor effects on renal function was found in 10 more patients compared to baseline showing decline in eGFR $<60 \mathrm{~mL} / \mathrm{min} / 1.73 \%(\mathrm{P}=0.0063)$. Side effects (Table 3) were reported in $14.8 \%$ of patients, with the most common being decline in eGFR to $<45 \mathrm{~mL} / \mathrm{min} / 1.73 \%(\mathrm{n}=5)$ and nasopharyngitis $(\mathrm{n}=3)$. There were no documented events of hypoglycemia. Ten patients $(12.3 \%)$ discontinued therapy with the most common reason being that they switched therapy to another SGLT-2 or other diabetes agent $(n=6)$ and only two $(20 \%)$ reported stopping due to genital mycotic infections and one $(10 \%)$ reported stopping due to nasopharyngitis.

Table 4 Reason for discontinuation of SGLT-2 inhibitor

\begin{tabular}{ll}
\hline Discontinuation reason $(\mathbf{n}=\mathbf{l} \mathbf{0})$ & $\mathbf{n}(\%)$ \\
\hline Cost/Insurance & $2(20 \%)$ \\
Genital Mycotic Infection & $2(20 \%)$ \\
Nasopharyngitis & $1(10 \%)$ \\
Switched therapy & $6(60 \%)$ \\
\hline
\end{tabular}

\section{Discussion}

Only 3 out of the 10 patients that discontinued therapy did so due to intolerance to side effects, specifically development of genital mycotic infection $(n=2)$ and nasopharyngitis $(n=1)$. While genital mycotic infections are a documented side effect and cause for discontinuation, nasopharyngitis is much less common and surprising to see in these results. ${ }^{10-13}$ Previously published data reports higher incidence of genital mycotic infections and much lower incidence of nasopharyngitis compared to our data. ${ }^{10,13}$ This could be due to the geographical location of the $\mathrm{PCMH}$ in the northeast where nasopharyngitis is common during certain times of the year and symptoms of nasopharyngitis can be easily mistaken for an allergy to pollen, another a common ailment in the northeast.

The mean time on an SGLT-2 inhibitor during our study period was 5months and it is known that the maximum effect can take up to 6months to be realized. ${ }^{12}$ Our study showed that these agents show efficacy, with an average HbA1c lowering of $-1.04 \%$, when used in a clinical environment in a PCMH. This is unique in that our mean change in $\mathrm{HbA} 1 \mathrm{c}$ after 3 months was better than previously published data. Monami et al published a met-analysis that showed a mean change in HbA1c of $-0.695 \%,{ }^{12}$ Rosentock et al. ${ }^{10}$ showed a similar lowering of HbA1c from baseline of $-0.76 \%$, and Berhan \& Barker ${ }^{11}$ reported a mean change in $\mathrm{HbA} 1 \mathrm{c}$ of $-0.78 \%$. Similarly, our results showed an average change in weight of -7 pounds $(-3.17 \mathrm{~kg})$ while other studies have shown weight changes of -5.7 pounds $(-2.6 \mathrm{~kg})$ and -1.3 pounds $(-0.59 \mathrm{~kg})$. In addition, our results showed a more pronounced lowering of SBP and DBP $(-10 \mathrm{mmHg}$ and $-4 \mathrm{mmHg}$ respectively) as compared to other published data. ${ }^{10,13}$

Hypoglycemia was not reported as a side effect or reason for discontinuation in our study population which is supported by their mechanism of action and overall incidence of hypoglycemia. ${ }^{8}$ Data was collected and reported on how many patients had a decline in renal function as measured by eGFR, since SGLT-2 inhibitors act on the kidneys and have to be renally dosed..$^{8,14}$ In addition, there were no documented cases of diabetic ketoacidosis (DKA) in this study 
population. Data related to the incidence of eGFR decline has not been previously published in a way that is meaningful to a PCMH clinician. Having a sense of how many of your patients will have declining renal function on an SGLT-2 inhibitor is invaluable to clinicians because they are better able to weigh the risks and benefits with patients before making a drug therapy decision.

\section{Limitations}

Our study has several limitations, including utilization of observational techniques. Although SGLT-2 inhibitors demonstrated favorable results, there is no establishment of cause and effect due to the retrospective nature of this study. Outcomes were demonstrated in a small patient population limited to one PCMH. A larger sample size in a variety of settings and locations may elicit different results. The study population was also predominantly Caucasian (79\%), middle aged to older adults (61years) with obesity (74.1\%). Additionally, the majority of participants were male (70.4\%). Lack of diversity shown in this population may hinder the applicability of these results to groups that do not fit this patient profile. The larger male representation could have also affected the incidence of certain adverse events such as genitourinary infections, which had a lower occurrence than expected.

A major limitation to our study was reliance on EMR for data collection. Only documented labs and reported side effects could be included, thus additional adverse events may have occurred that were not reported by patients or documented by clinicians. Some patient data was incomplete with all the required information for appropriate assessment of clinical efficacy of SGLT-2 inhibitors (e.g. HbA1c labs in the period of SGLT-2 use). Absence of necessary labs caused many patients to be excluded from the study to expand the analyzed population. Another drawback from EMR, was that eGFR values were not uniformly reported in patient records. For example, instead of indicating a specific eGFR value, eGFR $>60 \mathrm{~mL} / \mathrm{min} / 1.73 \%$ would be reported instead. This prevented more accurate statistical analysis of changes in eGFR to display the effects of SGLT-2 inhibitors on renal function.

Adherence was not evaluated, but it can be inferred that oral once daily dosing would cause less missed doses and better compliance compared to multiple daily doses and injectable therapy. Another uncontrollable factor was the development of infections and illnesses during the study period. These occurrences can exacerbate patients' glycemic control through stress, interruption in chronic therapy, hospitalizations, and steroid use. And given that this study took place in the northeastern region of the United States, allergies and the common cold are quite prevalent. Lastly, as the first SGLT-2 inhibitor approved, most patients observed were using canagliflozin as expected. Study outcomes can be possibly viewed as a more accurate representation of canagliflozin rather than a class effect.

\section{Conclusion}

This retrospective observational study in T2DM patients in a single PCMH demonstrated SGLT-2 inhibitor efficacy and safety showing significant improvements in $\mathrm{HbAlc}$, the number of patients to reach HbAlc goal $<7 \%$, weight loss, and blood pressure with minimal incidences of discontinuation and adverse events. SGLT-2 inhibitors exhibited a variety of favorable effects that would be beneficial in uncontrolled T2DM patients who have been unsuccessful with other diabetes drug classes. They are a safe and effective option for targeting multiple aspects of diabetes and the co-morbidities that accompany its progression.

\section{Acknowledgements}

None.

\section{Conflict of interest}

Author declares that there is no conflict of interest.

\section{Reference}

1. Centers for Disease Control and Prevention. National diabetes statistics report: estimates of diabetes and its burden in the United States. US Department of Health and Human Services, Atlanta, GA; 2014.

2. Saisho Y. Beta-cell dysfunction: its critical role in prevention and management of type 2 diabetes. World J Diabetes. 2015;6(1):109-124.

3. King P, Peacock I, Donnelly R. The UK prospective diabetes study (UKPDS): clinical and therapeutic implications for type 2 diabetes. $\mathrm{Br} J$ Clin Pharmacol. 1999;48(5):643-648.

4. Holman RR, Paul SK, Bethel MA, et al. 10year follow-up of intensive glucose control in type 2 diabetes. N Engl J Med. 2008;359:1577-1589.

5. Association AD. Standards of medical care in diabetes 2015. Diabetes Care. 2015;38:S1-S93.

6. Garber AJ, Abrahamson MJ, Barzilay JI, et al. Consensus statement by the American Association of Clinical Endocrinologists and American College of Endocrinology on the comprehensive type 2 diabetes management algorithm 2016 executive summary. Endocr Pract. 2016;22(1):84-113.

7. Inzucchi SE, Bergenstal RM, Buse JB, et al. Management of hyperglycemia in type 2 diabetes: a patient-centered approach: position statement of the American Diabetes Association (ADA) and the European Association for the Study of Diabetes (EASD). Diabetes Care. 2012;35(6):1364-1379.

8. Neumiller JJ, White JR, Campbell RK. Sodium-glucose co-transport inhibitors: progress and therapeutic potential in type 2 diabetes mellitus. Drugs. 2010;70(4):377-385.

9. Abdul-Ghani MA, Norton L, Defronzo RA. Role of sodium-glucose cotransporter 2 (SGLT 2) inhibitors in the treatment of type 2 diabetes. Endocr Rev. 2011;32(4):515-531.

10. Rosenstock J, Aggarwal N, Polidori D, et al. Dose-ranging effects of canagliflozin, a sodium-glucose cotransporter 2 inhibitor, as addon to metformin in subjects with type 2 diabetes. Diabetes Care. 2012;35(6):1232-1238

11. Berhan A, Barker A. Sodium glucose co-transport 2 inhibitors in the treatment of type 2 diabetes mellitus: a meta-analysis of randomized double-blind controlled trials. BMC Endocr Disord. 2013;13:58.

12. Monami M, Nardini C, Mannucci E. Efficacy and safety of sodium glucose co-transport-2 inhibitors in type 2 diabetes: a meta-analysis of randomized clinical trials. Diabetes Obes Metab. 2014;16(5):457-466.

13. Musso G, Gambino R, Cassader M, et al. A novel approach to control hyperglycemia in type 2 diabetes: sodium glucose co-transport (SGLT) inhibitors: systematic review and meta-analysis of randomized trials. Ann Med. 2012;44(4):375-393.

14. Sha S, Devineni D, Ghosh A, et al. Canagliflozin, a novel inhibitor of sodium glucose co-transporter 2, dose dependently reduces calculated renal threshold for glucose excretion and increases urinary glucose excretion in healthy subjects. Diabetes Obes Metab. 2011;13(7):669-672. 